\title{
The sharp bound of the third Hankel determinant for functions of bounded turning
}

\section{Bogumiła Kowalczyk $^{1} \cdot$ Adam Lecko $^{1}$}

Received: 20 June 2021 / Accepted: 23 August 2021 / Published online: 4 September 2021

(C) The Author(s) 2021

\section{Abstract}

We find the sharp bound for the third Hankel determinant

$$
H_{3,1}(f):=\left|\begin{array}{lll}
a_{1} & a_{2} & a_{3} \\
a_{2} & a_{3} & a_{4} \\
a_{3} & a_{4} & a_{5}
\end{array}\right|
$$

for analytic functions $f$ with $a_{n}:=f^{(n)}(0) / n !, n \in \mathbb{N}, a_{1}:=1$, such that

$$
\operatorname{Re} f^{\prime}(z)>0, \quad z \in \mathbb{D}:=\{z \in \mathbb{C}:|z|<1\} .
$$

Keywords Univalent function - Function of bounded turning · Hankel determinant · Carathéodory function · Coefficient

Mathematics Subject Classification $30 \mathrm{C} 45 \cdot 30 \mathrm{C} 50$

\section{Introduction}

Let $\mathcal{H}$ be the class of all analytic functions in $\mathbb{D}:=\{z \in \mathbb{C}:|z|<1\}$ and $\mathcal{A}$ be the subclass of functions $f$ normalized by $f(0)=0, f^{\prime}(0)=1$, i.e., of the form

Adam Lecko

alecko@matman.uwm.edu.pl

Bogumiła Kowalczyk

b.kowalczyk@matman.uwm.edu.pl

1 Department of Complex Analysis, Faculty of Mathematics and Computer Science, University of Warmia and Mazury in Olsztyn, ul. Słoneczna 54, 10-710 Olsztyn, Poland 


$$
f(z)=\sum_{n=1}^{\infty} a_{n} z^{n}, \quad a_{1}:=1, z \in \mathbb{D},
$$

and let $\mathcal{S}$ be the subclass of $\mathcal{A}$ of univalent functions. We denote by $\mathcal{P}^{\prime}$ the subfamily of $\mathcal{A}$ of all functions $f$ such that

$$
\operatorname{Re} f^{\prime}(z)>0, \quad z \in \mathbb{D} .
$$

Functions in $\mathcal{P}^{\prime}$ are called functions of bounded turning (cf. [6, Vol. I, p. 101]), and in recent times many authors have denoted this class of functions by $\mathcal{R}$.

It is well-known [1] (cf. [6, Vol. I, p. 88]) that $\mathcal{P}^{\prime} \subset \mathcal{S}$, and is a fundamental subfamily of univalent functions which has been extensively studied by many authors, e.g., [17, 18].

For $q, n \in \mathbb{N}$, the Hankel determinant $H_{q, n}(f)$ of function $f \in \mathcal{A}$ of the form (1) is defined as

$$
H_{q, n}(f):=\left|\begin{array}{cccc}
a_{n} & a_{n+1} & \cdots & a_{n+q-1} \\
a_{n+1} & a_{n+2} & \cdots & a_{n+q} \\
\vdots & \vdots & \vdots & \vdots \\
a_{n+q-1} & a_{n+q} & \cdots & a_{n+2(q-1)}
\end{array}\right| .
$$

General results for Hankel determinants of any degree with their applications can be found in $[4,20,21,23]$. For a subfamily $\mathcal{F}$ of $\mathcal{A}, q$ and $n$, computing the upper bound of $H_{q, n}(f)$ represents an interesting and important problem. Hayman [7] examined the second Hankel determinant of mean for univalent functions. Recently, many authors examined the second Hankel determinant $H_{2,2}(f)=a_{2} a_{4}-a_{3}^{2}$ (see e.g., $[5,15]$ with further references). The problem of finding sharp estimating of the third Hankel determinant

$$
H_{3,1}(f)=\left|\begin{array}{lll}
a_{1} & a_{1} & a_{3} \\
a_{2} & a_{3} & a_{4} \\
a_{3} & a_{4} & a_{5}
\end{array}\right|=a_{3}\left(a_{2} a_{4}-a_{3}^{2}\right)-a_{4}\left(a_{4}-a_{2} a_{3}\right)+a_{5}\left(a_{3}-a_{2}^{2}\right)
$$

is technically much more difficult, and few sharp bounds have been obtained. However, sharp bounds of $\left|H_{3,1}(f)\right|$ have been obtained for convex functions [12], starlike functions of order $1 / 2$ [14], and functions $f \in \mathcal{A}$ which satisfy the condition $\operatorname{Re} f(z) / z>\alpha, z \in \mathbb{D}$, in the case $\alpha=0$ and $\alpha=1 / 2$ [11], and functions $f \in \mathcal{A}$ such that $\left|(z / f(z))^{2}-1\right|<1$ for $z \in \mathbb{D}[19]$.

When $f \in \mathcal{P}^{\prime}$ Janteng et al. [9] showed that $\left|H_{3,1}(f)\right| \leq 439 / 540=0.81296$. . which is not sharp, and originally proved in [2]. The proof used (3) and sharp estimates of expressions contained in (3), namely $\left|a_{2} a_{4}-a_{3}^{2}\right| \leq 4 / 9$ found in [8], $\left|a_{2} a_{3}-a_{4}\right| \leq 1 / 2$ in [2] and reproved in [9], $\left|a_{3}-a_{2}^{2}\right| \leq 2 / 3$ cited from [2] (see also [10], where this result was proved earlier) and $\left|a_{n}\right| \leq 2 / n$ found in [18]. We also note that proofs in [2] contained some gaps, and new proofs were obtained in [9]. The aforementioned estimate of $\left|H_{3,1}(f)\right|$ in $\mathcal{P}^{\prime}$ was improved in [24], where the author shown that $\left|H_{3,1}(f)\right| \leq 41 / 60=0.683 \ldots$, which is also not sharp, and 
discussed $H_{3,1}(f)$ for subclasses $\mathcal{P}^{\prime(2)}$ and $\mathcal{P}^{\prime(3)}$ of $\mathcal{P}^{\prime}$ consisting of 2-fold and 3 -fold symmetric functions, respectively, and obtained the following sharp bounds: $\left|H_{3,1}(f)\right| \leq 2 \sqrt{6} / 45=0.108 \ldots$ and $\left|H_{3,1}(f)\right| \leq 1 / 4$ for $f$ in $\mathcal{P}^{\prime(2)}$ and $\mathcal{P}^{\prime(3)}$, respectively.

In this paper, we show that $\left|H_{3,1}(f)\right| \leq 1 / 4$ for $f \in \mathcal{P}^{\prime}$ and that the inequality is sharp.

Since the class $\mathcal{P}^{\prime}$ can be represented using the Carathéodory class $\mathcal{P}$, i.e., the class of functions $p \in \mathcal{H}$ of the form

$$
p(z)=1+\sum_{n=1}^{\infty} c_{n} z^{n}, \quad z \in \mathbb{D},
$$

having a positive real part in $\mathbb{D}$, the coefficients of functions in $\mathcal{P}^{\prime}$ can be expressed as coefficients of functions in $\mathcal{P}$. We then obtain the upper bound of $\left|H_{3,1}(f)\right|$, basing our analysis on the well-known formulas of coefficient $c_{2}$ (e.g., [22, p. 166]), the formula $c_{3}$ due to Libera and Zlotkiewicz [16, 17], and the formula for $c_{4}$ recently found in [13].

\section{Main result}

The basis for proof of the main result is the following lemma. It contains the well known formula for $c_{2}$ (e.g., [22, p. 166]). the formula for $c_{3}$ due to Libera and Zlotkiewicz [16, 17] and the formula for $c_{4}$ found in [13].

Lemma 1 If $p \in \mathcal{P}$ is of the form (4) with $c_{1} \geq 0$, then

$$
\begin{gathered}
c_{1}=2 \zeta_{1}, \\
c_{2}=2 \zeta_{1}^{2}+2\left(1-\zeta_{1}^{2}\right) \zeta_{2}, \\
c_{3}=2 \zeta_{1}^{3}+4\left(1-\zeta_{1}^{2}\right) \zeta_{1} \zeta_{2}-2\left(1-\zeta_{1}^{2}\right) \zeta_{1} \zeta_{2}^{2}+2\left(1-\zeta_{1}^{2}\right)\left(1-\left|\zeta_{2}\right|^{2}\right) \zeta_{3}
\end{gathered}
$$

and

$$
\begin{aligned}
c_{4}= & 2 \zeta_{1}^{4}+2\left(1-\zeta_{1}^{2}\right) \zeta_{2}\left(\zeta_{1}^{2} \zeta_{2}^{2}-3 \zeta_{1}^{2} \zeta_{2}+3 \zeta_{1}^{2}+\zeta_{2}\right) \\
& +2\left(1-\zeta_{1}^{2}\right)\left(1-\left|\zeta_{2}\right|^{2}\right) \zeta_{3}\left(2 \zeta_{1}-2 \zeta_{1} \zeta_{2}-\overline{\zeta_{2} \zeta_{3}}\right) \\
& +2\left(1-\zeta_{1}^{2}\right)\left(1-\left|\zeta_{2}\right|^{2}\right)\left(1-\left|\zeta_{3}\right|^{2}\right) \zeta_{4},
\end{aligned}
$$

for some $\zeta_{1} \in[0,1]$ and $\zeta_{2}, \zeta_{3}, \zeta_{4} \in \overline{\mathbb{D}}$.

We now prove the main theorem of this paper. 


\section{Theorem 1}

$$
\max \left\{\left|H_{3,1}(f)\right|: f \in \mathcal{P}^{\prime}\right\}=\frac{1}{4}
$$

with extreme function $f_{0} \in \mathcal{P}^{\prime}$ given by

$$
f_{0}^{\prime}(z):=\frac{1-z^{3}}{1+z^{3}}, \quad z \in \mathbb{D} .
$$

Proof Let $f \in \mathcal{P}^{\prime}$ and be given by (1). Then by (2),

$$
f^{\prime}(z)=p(z), \quad z \in \mathbb{D},
$$

for some function $p \in \mathcal{P}$ of the form (4). Since both the classes $\mathcal{P}^{\prime}$ and $\mathcal{P}$ and the functional $H_{3,1}(f)$ are invariant under the rotations, we may assume that $c_{1} \in[0,2]$ ( [3], see also [6, Vol. I, p. 80, Theorem 3]), i.e., in view of (5) that $\zeta_{1} \in[0,1]$.

Substituting (1) and (4) into (11) and equating coefficients, we obtain

$$
a_{2}=\frac{1}{2} c_{1}, \quad a_{3}=\frac{1}{3} c_{2}, \quad a_{4}=\frac{1}{4} c_{3}, \quad a_{5}=\frac{1}{5} c_{4} .
$$

Hence, by (3), we have

$$
H_{3,1}(f)=\frac{1}{2160}\left(180 c_{1} c_{2} c_{3}-80 c_{2}^{3}-135 c_{3}^{2}+144 c_{2} c_{4}-108 c_{1}^{2} c_{4}\right) .
$$

Using (5)-(8) by straightforward algebraic computation we obtain

$$
\begin{aligned}
180 c_{1} c_{2} c_{3}= & 1440\left[\zeta_{1}^{6}+\left(1-\zeta_{1}^{2}\right)\left(3-\zeta_{2}\right) \zeta_{1}^{4} \zeta_{2}\right. \\
& +\left(1-\zeta_{1}^{2}\right)^{2}\left(2-\zeta_{2}\right) \zeta_{1}^{2} \zeta_{2}^{2}+\left(1-\zeta_{1}^{2}\right)\left(1-\left|\zeta_{2}\right|^{2}\right) \zeta_{1}^{3} \zeta_{3} \\
& \left.+\left(1-\zeta_{1}^{2}\right)^{2}\left(1-\left|\zeta_{2}\right|^{2}\right) \zeta_{1} \zeta_{2} \zeta_{3}\right], \\
-80 c_{2}^{3}= & -640\left[\zeta_{1}^{6}+3\left(1-\zeta_{1}^{2}\right) \zeta_{1}^{4} \zeta_{2}+3\left(1-\zeta_{1}^{2}\right)^{2} \zeta_{1}^{2} \zeta_{2}^{2}+\left(1-\zeta_{1}^{2}\right)^{3} \zeta_{2}^{3}\right], \\
-135 c_{3}^{2}= & -540\left[\zeta_{1}^{6}+2\left(1-\zeta_{1}^{2}\right)\left(2-\zeta_{2}\right) \zeta_{1}^{4} \zeta_{2}+\left(1-\zeta_{1}^{2}\right)^{2}\left(2-\zeta_{2}\right)^{2} \zeta_{1}^{2} \zeta_{2}^{2}\right. \\
& +2\left(1-\zeta_{1}^{2}\right)\left(1-\left|\zeta_{2}\right|^{2}\right) \zeta_{1}^{3} \zeta_{3}+2\left(1-\zeta_{1}^{2}\right)^{2}\left(1-\left|\zeta_{2}\right|^{2}\right)\left(2-\zeta_{2}\right) \zeta_{1} \zeta_{2} \zeta_{3} \\
& \left.+\left(1-\zeta_{1}^{2}\right)^{2}\left(1-\left|\zeta_{2}\right|^{2}\right)^{2} \zeta_{3}^{2}\right]
\end{aligned}
$$

and 


$$
\begin{aligned}
& 144 c_{2} c_{4}-108 c_{1}^{2} c_{4} \\
& =288\left[-\zeta_{1}^{6}+\left(1-\zeta_{1}^{2}\right)\left(-\zeta_{1}^{2} \zeta_{2}-\zeta_{1}^{2} \zeta_{2}^{3}+3 \zeta_{1}^{2} \zeta_{2}^{2}-\zeta_{2}^{2}\right) \zeta_{1}^{2}\right. \\
& +2\left(1-\zeta_{1}^{2}\right)^{2}\left(3 \zeta_{1}^{2} \zeta_{2}^{2}+\zeta_{1}^{2} \zeta_{2}^{4}-3 \zeta_{1}^{2} \zeta_{2}^{3}+\zeta_{2}^{3}\right) \\
& -\left(1-\zeta_{1}^{2}\right)\left(1-\left|\zeta_{2}\right|^{2}\right)\left(2 \zeta_{1} \zeta_{3}-2 \zeta_{1} \zeta_{2} \zeta_{3}-\overline{\zeta_{2}} \zeta_{3}^{2}\right) \zeta_{1}^{2} \\
& +2\left(1-\zeta_{1}^{2}\right)^{2}\left(1-\left|\zeta_{2}\right|^{2}\right)\left(2 \zeta_{1} \zeta_{2} \zeta_{3}-2 \zeta_{1} \zeta_{2}^{2} \zeta_{3}-\left|\zeta_{2}\right|^{2} \zeta_{3}^{2}\right) \\
& -\left(1-\zeta_{1}^{2}\right)\left(1-\left|\zeta_{2}\right|^{2}\right)\left(1-\left|\zeta_{3}\right|^{2}\right) \zeta_{1}^{2} \zeta_{4} \\
& \left.+2\left(1-\zeta_{1}^{2}\right)^{2}\left(1-\left|\zeta_{2}\right|^{2}\right)\left(1-\left|\zeta_{3}\right|^{2}\right) \zeta_{2} \zeta_{4}\right] \text {. }
\end{aligned}
$$

Substituting the above expression into (12), we obtain

$$
H_{3,1}(f)=\frac{1}{540}\left[\gamma_{1}\left(\zeta_{1}, \zeta_{2}\right)+\gamma_{2}\left(\zeta_{1}, \zeta_{2}\right) \zeta_{3}+\gamma_{3}\left(\zeta_{1}, \zeta_{2}\right) \zeta_{3}^{2}+\gamma_{4}\left(\zeta_{1}, \zeta_{2}, \zeta_{3}\right) \zeta_{4}\right]
$$

where for $\zeta_{1} \in[0,1]$ and $\zeta_{2}, \zeta_{3}, \zeta_{4} \in \overline{\mathbb{D}}$,

$$
\begin{aligned}
\gamma_{1}\left(\zeta_{1}, \zeta_{2}\right):= & -7 \zeta_{1}^{6}-12\left(1-\zeta_{1}^{2}\right) \zeta_{1}^{4} \zeta_{2}+6\left(1-\zeta_{1}^{2}\right)\left(10-\zeta_{1}^{2}\right) \zeta_{1}^{2} \zeta_{2}^{2} \\
& +4\left(1-\zeta_{1}^{2}\right)\left(5 \zeta_{1}^{4}-19 \zeta_{1}^{2}-4\right) \zeta_{2}^{3}+9\left(1-\zeta_{1}^{2}\right)^{2} \zeta_{1}^{2} \zeta_{2}^{4}, \\
\gamma_{2}\left(\zeta_{1}, \zeta_{2}\right):= & -18\left(1-\zeta_{1}^{2}\right)\left(1-\left|\zeta_{2}\right|^{2}\right)\left[3 \zeta_{1}^{3}-\left(2 \zeta_{1}^{2}+6\right) \zeta_{1} \zeta_{2}+\left(1-\zeta_{1}^{2}\right) \zeta_{1} \zeta_{2}^{2}\right], \\
\gamma_{3}\left(\zeta_{1}, \zeta_{2}\right):= & 9\left(1-\zeta_{1}^{2}\right)\left(1-\left|\zeta_{2}\right|^{2}\right)\left[8 \zeta_{1}^{2} \overline{\zeta_{2}}-\left(1-\zeta_{1}^{2}\right)\left(\left|\zeta_{2}\right|^{2}+15\right)\right]
\end{aligned}
$$

and

$$
\gamma_{4}\left(\zeta_{1}, \zeta_{2}, \zeta_{3}\right):=72\left(1-\zeta_{1}^{2}\right)\left(1-\left|\zeta_{2}\right|^{2}\right)\left(1-\left|\zeta_{3}\right|^{2}\right)\left[-\zeta_{1}^{2}+2\left(1-\zeta_{1}^{2}\right) \zeta_{2}\right] .
$$

Since $\left|\zeta_{4}\right| \leq 1$, from (13) we obtain

$$
\begin{aligned}
\left|H_{3,1}(f)\right| \leq & \frac{1}{540}\left[\left|\gamma_{1}\left(\zeta_{1}, \zeta_{2}\right)\right|+\left|\gamma_{2}\left(\zeta_{1}, \zeta_{2}\right)\right|\left|\zeta_{3}\right|\right. \\
& \left.+\left|\gamma_{3}\left(\zeta_{1}, \zeta_{2}\right)\right|\left|\zeta_{3}\right|^{2}+\left|\gamma_{4}\left(\zeta_{1}, \zeta_{2}, \zeta_{3}\right)\right|\right] \\
\leq & \frac{1}{540} \max \left\{\Phi(x, y, u):(x, y, u) \in D:=[0,1]^{3}\right\}
\end{aligned}
$$

where

$$
\Phi(x, y, u):=\varphi_{1}(x, y)+\varphi_{4}(x, y)+\varphi_{2}(x, y) u+\left[\varphi_{3}(x, y)-\varphi_{4}(x, y)\right] u^{2},
$$

with 


$$
\begin{aligned}
& \varphi_{1}(x, y):= 7 x^{6}+12\left(1-x^{2}\right) x^{4} y+6\left(1-x^{2}\right)\left(10-x^{2}\right) x^{2} y^{2} \\
&+4\left(1-x^{2}\right)\left(5 x^{2}+1\right)\left(4-x^{2}\right) y^{3}+9\left(1-x^{2}\right)^{2} x^{2} y^{4}, \\
& \varphi_{2}(x, y):= 18 x\left[3 x^{2}+2\left(3+x^{2}\right) y+\left(1-x^{2}\right) y^{2}\right]\left(1-x^{2}\right)\left(1-y^{2}\right), \\
& \varphi_{3}(x, y):=9\left[8 x^{2} y+\left(1-x^{2}\right)\left(y^{2}+15\right)\right]\left(1-x^{2}\right)\left(1-y^{2}\right),
\end{aligned}
$$

and

$$
\varphi_{4}(x, y):=72\left[x^{2}+2\left(1-x^{2}\right) y\right]\left(1-x^{2}\right)\left(1-y^{2}\right) .
$$

We will now show that

$$
\max \{\Phi(x, y, u):(x, y, u) \in D\}=135 .
$$

A. On the vertices of $D$, we have

$$
\begin{aligned}
& \Phi(0,0,0)=0, \quad \Phi(0,0,1)=135, \quad \Phi(0,1,0)=\Phi(0,1,1)=16, \\
& \Phi(1,0,0)=\Phi(1,0,1)=\Phi(1,1,0)=\Phi(1,1,1)=7 .
\end{aligned}
$$

B. We next consider the edges of $D$.

1. $y=0, u=0$. Then

$$
\begin{aligned}
& \Phi(x, 0,0)=7 x^{6}-72 x^{4}+72 x^{2} \leq \Phi\left(x_{1}, 0,0\right) \\
& \quad=\frac{96}{49}(17 \sqrt{102}-162) \approx 18.988, \quad x \in(0,1),
\end{aligned}
$$

where

$$
x_{1}:=\sqrt{\frac{2}{7}(12-\sqrt{102})} \approx 0.737 .
$$

2. $y=1, u=0$. Then,

$$
\begin{aligned}
& \Phi(x, 1,0)=30 x^{6}-168 x^{4}+129 x^{2}+16 \leq \Phi\left(x_{2}, 1,0\right) \\
& =\frac{1}{225}(923 \sqrt{1846}-30028) \approx 42.795, \quad x \in(0,1),
\end{aligned}
$$

where

$$
x_{2}:=\sqrt{\frac{56-\sqrt{1846}}{30}} \approx 0.659 .
$$

3. $x=0, u=0$. Then,

$$
\Phi(0, y, 0)=-128 y^{3}+144 y \leq \Phi\left(0, \frac{\sqrt{6}}{4}, 0\right)=24 \sqrt{6} \approx 58.788, \quad y \in(0,1) .
$$

4. $x=1, u=0$. Then, 


$$
\Phi(1, y, 0)=7, \quad y \in[0,1]
$$

5. $y=0, u=1$. Then,

$$
\Phi(x, 0,1)=7 x^{6}-54 x^{5}+135 x^{4}+54 x^{3}-270 x^{2}+135 \leq 135, \quad x \in(0,1),
$$

which is equivalent to

$$
7 x^{4}-54 x^{3}+135 x^{2}+54 x-270 \leq 0, \quad x \in[0,1],
$$

and is easily seen to be true.

6. $y=1, u=1$. Since $\Phi(x, 1,1)=\Phi(x, 1,0)$ for $x \in(0,1)$, which reduces to case 2 .

7. $x=0, u=1$. Then,

$$
\Phi(0, y, 1)=-9 y^{4}+16 y^{3}-126 y^{2}+135 \leq 135, \quad y \in(0,1),
$$

which is equivalent to

$$
y^{2}\left(-9 y^{2}+16 y-126\right) \leq 0, \quad y \in(0,1),
$$

and again is evidently true.

8. $x=1, u=1$. Then,

$$
\Phi(1, y, 1)=7, \quad y \in(0,1) .
$$

9. $x=0, y=0$. Then,

$$
\Phi(0,0, u)=135 u^{2} \leq 135, \quad u \in(0,1) .
$$

10. $x=0, y=1$. Then,

$$
\Phi(0,1, u)=16, \quad u \in(0,1) .
$$

11. $x=1, y=0$. Then,

$$
\Phi(1,0, u)=7, \quad u \in(0,1) .
$$

12. $x=1, y=1$. Then,

$$
\Phi(1,1, u)=7, \quad u \in(0,1) .
$$

C. We consider now the faces of $D$.

1. $x=1$. Then,

$$
\Phi(1, y, u)=7, \quad y, u \in(0,1) .
$$

2. $x=0$. Then,

$$
\Phi(0, y, u)=114 y-128 y^{3}+9\left(1-y^{2}\right)(1-y)(15-y) u^{2}=: G_{1}(y, u), \quad y, u \in(0,1) .
$$

Clearly $G_{1}$ has no critical point in $(0,1) \times(0,1)$. 
3. $y=1$. Since $\Phi(x, 1, u)=\Phi(x, 1,0)$ for $x, u \in(0,1)$, this case reduces to the case B.2.

4. $y=0$. Then,

$$
\begin{aligned}
& \Phi(x, 0, u)=7 x^{6}-72 x^{4}+72 x^{2}+54 x^{3}\left(1-x^{2}\right) u+9\left(1-x^{2}\right)\left(15-23 x^{2}\right) u^{2} \\
& \quad=: G_{2}(x, u), \quad x, u \in(0,1) .
\end{aligned}
$$

We have

$$
\frac{\partial G_{2}}{\partial u}=54 x^{3}\left(1-x^{2}\right)+18\left(1-x^{2}\right)\left(15-23 x^{2}\right) u, \quad x, u \in(0,1) .
$$

Clearly $\partial G_{2} / \partial u \neq 0$ for $x=\sqrt{15 / 23}$. For $x \neq \sqrt{15 / 23}$ we see that $\partial G_{2} / \partial u=0$ if, and only if,

$$
u=\frac{3 x^{3}}{23 x^{2}-15}=: u(x) .
$$

Note that $u(x) \in(0,1)$ if, and only if, $23 x^{2}-15>0$ and $3 x^{3}-23 x^{2}+15<0$, i.e., if, and only if, $x \in\left(x_{3}, 1\right)$, where $x_{3} \approx 0.857$. Since

$$
\frac{\partial G_{2}}{\partial x}(x, u(x))=0
$$

if, and only if,

$$
1840 x^{8}-27360 x^{6}+46176 x^{4}-27360 x^{2}+5400=0
$$

and the last equation has no root in $\left(x_{3}, 1\right)$ (all real roots are the following: $x \approx$ \pm 0.68045 and $x \approx \pm 3.60968)$ ), we conclude that $G_{2}$ has no critical point.

5. $u=0$. We have

$$
\begin{aligned}
\Phi(x, y, 0)= & 7 x^{6}-72 x^{4}+72 x^{2}+\left(-12 x^{6}+156 x^{4}-288 x^{2}+144\right) y \\
& +\left(6 x^{6}+6 x^{4}-12 x^{2}\right) y^{2}+\left(20 x^{6}-240 x^{4}+348 x^{2}-128\right) y^{3} \\
& +\left(9 x^{6}-18 x^{4}-9 x^{2}\right) y^{4}=: G_{3}(x, y), \quad x, y \in(0,1) .
\end{aligned}
$$

Now set $t:=x^{2} \in(0,1)$. Then $\partial G_{3} / \partial y=0$ if, and only if,

$$
(y+1)^{2}(1-3 y) t^{2}+\left(-12-2 y+55 y^{2}+3 y^{3}\right) t+12-32 y^{2}=0,
$$

and $\partial G_{3} / \partial t=0$ if, and only if,

$$
\begin{aligned}
& \left(7-12 y+6 y^{2}+20 y^{3}+9 y^{4}\right) t^{2}+\left(-48+104 y-4 y^{2}-160 y^{3}-12 y^{4}\right) t \\
& +24-96 y-4 y^{2}+116 y^{3}+3 y^{4}=0 .
\end{aligned}
$$

(a) Let $y=1 / 3$. Then, the Eq. (14) reduces to $t=76 / 58>1$.

(b) Suppose that $y \neq 1 / 3$. Then by (14), 


$$
\Delta:=9 y^{6}-54 y^{5}+2373 y^{4}-276 y^{3}-948 y^{2}+96 y+96>0
$$

for $y \in(0,1) \backslash\{1 / 3\}$ (all real roots of $\Delta=0$ are: $y \approx-0.5108$ and $y \approx-0.3278$ ).

Let

$$
t_{1,2}:=\frac{12+2 y-55 y^{2}-3 y^{3} \mp \sqrt{\Delta}}{2(y+1)^{2}(1-3 y)} .
$$

(i) Observe that $t_{1}>1$. Indeed, for $0<y<1 / 3$ and $1 / 3<y<1$ this inequality is equivalent to the obviously true inequalities

$$
(y+1)^{2}(1-3 y)\left(72 y^{2}-12 y+4\right)>0
$$

and

$$
(y+1)^{2}(1-3 y)\left(72 y^{2}-12 y+4\right)<0,
$$

respectively.

(ii) Since $12+2 y-55 y^{2}-3 y^{3}>0$ for $0<y<1 / 3$, then $t_{2}>0$. Note that the inequality $t_{2}>0$ is false for $y \in\left(1 / 3, y_{1}\right)$, where $y_{1} \approx 0.479$ is a unique positive solution of $12+2 y-55 y^{2}-3 y^{3}=0$. For $y \in\left(y_{1}, 1\right)$ the inequality $t_{2}>0$ is equivalent to

$$
(y+1)^{2}(3 y-1)\left(8 y^{2}-3\right)>0,
$$

which is true for $y \in(\sqrt{6} / 4,1)$.

Further, the inequality $t_{2}<1$ which is equivalent to $\sqrt{\Delta}<-10-4 y+45 y^{2}-$ $3 y^{3}$ is evidently false for $y \in(0,1 / 3)$, and is true when $y \in\left(1 / 3, y_{2}\right)$, where $y_{2} \approx$ 0.528 is a unique root in $(0,1)$ of the equation $-10-4 y+45 y^{2}-3 y^{3}=0$. For $y \in\left(y_{2}, 1\right)$ the inequality $t_{2}<1$ is equivalent to

$$
(y+1)^{2}(3 y-1)\left(18 y^{2}-3 y+1\right)>0,
$$

which is also true.

Summarising $t_{2} \in(0,1)$ if, and only if, $y \in(\sqrt{6} / 4,1)$, and substituting $t_{2}$ into (15) we obtain

$$
\begin{aligned}
& \left(7-12 y+6 y^{2}+20 y^{3}+9 y^{4}\right) t_{2}^{2}+\left(-48+104 y+4 y^{2}-160 y^{3}-12 y^{4}\right) t_{2} \\
& +24-96 y-4 y^{2}+116 y^{3}+3 y^{4}=0,
\end{aligned}
$$

which is equivalent to 


$$
\begin{aligned}
L(y):= & 54 y^{10}+180 y^{9}+4194 y^{8}+30672 y^{7}+27630 y^{6}-46356 y^{5} \\
& \quad+13754 y^{4}+4360 y^{3}-7000 y^{2}+1008 y+624 \\
= & \left(-18 y^{7}+30 y^{6}+600 y^{5}+660 y^{4}-702 y^{3}+394 y^{2}-44 y-72\right) \sqrt{\Delta}=: R(y) .
\end{aligned}
$$

Note that $L(y)>0$ for $y \in\left(y_{3}, 1\right)$ and $R(y)>0$ for $y \in\left(y_{4}, 1\right)$, where $y_{3} \approx 0.5782$ and $y_{4} \approx 0.5555$ are roots of $L$ and $R / \sqrt{\Delta}$, respectively. Therefore both sides of (17) are positive for $y \in(\sqrt{6} / 4,1)$. Thus (17) is equivalent to

$$
\begin{aligned}
& L^{2}(y)-R^{2}(y)=(y+1)^{4}(3 y-1)^{2}\left(5184 y^{13}-34560 y^{12}+791424 y^{11}\right. \\
& \quad+6098976 y^{10}+3934080 y^{9}-14940000 y^{8}-1216512 y^{7}+6150528 y^{6} \\
& \left.\quad-4316544 y^{5}+2653696 y^{4}-1009152 y^{3}+743424 y^{2}-64512 y-108288\right)=0 .
\end{aligned}
$$

A numerical computation shows that all real solution of the above equation are as follow:

$$
\begin{aligned}
& y=-1, \quad y=\frac{1}{3}, \quad y \approx-4.2848, \quad y \approx-2.3680, \quad y \approx-1.0721, \\
& y \approx-0.2646, \quad y \approx 0.5478, \quad y \approx 0.5613, \quad y \approx 1.0977
\end{aligned}
$$

Thus, the Eq. (18), so (16) has no solution in $(\sqrt{6} / 4,1)$, and so , $G_{3}$ has no critical point.

6. $u=1$. We have

$$
\begin{aligned}
\Phi(x, y, 1)= & 7 x^{6}-54 x^{5}+135 x^{4}+54 x^{3}-270 x^{2}+135 \\
& +\left(-12 x^{6}-36 x^{5}-60 x^{4}-72 x^{3}+72 x^{2}+108 x\right) y \\
& +\left(6 x^{6}+72 x^{5}-192 x^{4}-90 x^{3}+312 x^{2}+18 x-126\right) y^{2} \\
& +\left(20 x^{6}+36 x^{5}-24 x^{4}+72 x^{3}-12 x^{2}-108 x+16\right) y^{3} \\
& +\left(9 x^{6}-18 x^{5}-27 x^{4}+36 x^{3}+27 x^{2}-18 x-9\right) y^{4}=: G_{4}(x, y)
\end{aligned}
$$

for $x, y \in(0,1)$. Then, $\partial G_{4} / \partial x=0$ if, and only if, 


$$
\begin{aligned}
& 7 x^{5}-45 x^{4}+90 x^{3}+27 x^{2}-90 x \\
& +\left(-12 x^{5}-30 x^{4}-40 x^{3}-36 x^{2}+24 x+108\right) y \\
& +\left(6 x^{5}+60 x^{4}-128 x^{3}-45 x^{2}+104 x+3\right) y^{2} \\
& +\left(20 x^{5}+30 x^{4}-16 x^{3}+36 x^{2}-4 x-18\right) y^{3} \\
& +\left(9 x^{5}-15 x^{4}-18 x^{3}+18 x^{2}+9 x-3\right) y^{4}=0
\end{aligned}
$$

and $\partial G_{4} / \partial y=0$ if, and only if,

$$
\begin{aligned}
& -\left(1-x^{2}\right)\left[-x^{4}-3 x^{3}-6 x^{2}-9 x+\left(x^{4}+12 x^{3}-31 x^{2}-3 x+21\right) y\right. \\
& \left.\quad+\left(5 x^{4}+9 x^{3}-x^{2}+27 x-4\right) y^{2}+\left(3 x^{4}-6 x^{3}-6 x^{2}+6 x+3\right) y^{3}\right]=0 .
\end{aligned}
$$

Another numerical computation shows that all real solutions of the system of Eqs. (19) and (20) are as follow:

$$
\begin{aligned}
& (x=0, y=0), \quad(x \approx-0.9436, y \approx 0.9093), \quad(x \approx-0.9005, y \approx 0.2632), \\
& (x \approx-0.7232, y \approx-18.8987), \quad(x \approx-0.4941, y \approx 0.9093), \\
& (x \approx 1.2834, y \approx 16.4015), \quad(x \approx 5.4293, y \approx-3.4718, \\
& (x \approx 6.1058, y \approx-3.0055), \quad(x \approx 8.9567, y \approx 0.3360) .
\end{aligned}
$$

Therefore, $G_{4}$ has no critical point.

D. It remains to consider the interior of $D$, i.e., $(0,1)^{3}$. We have

$$
\begin{aligned}
\Phi(x, y, u)= & \varphi_{1}(x, y)+\varphi_{4}(x, y)+\varphi_{2}(x, y) u+\left[\varphi_{3}(x, y)-\varphi_{4}(x, y)\right] u^{2} \\
& 7 x^{6}+12 x^{2}\left(1-x^{2}\right) y+6 x^{2}\left(1-x^{2}\right)\left(10-x^{2}\right) y^{2} \\
& +4\left(1-x^{2}\right)\left(1+5 x^{2}\right)\left(4-x^{2}\right) y^{3}+9 x^{2}\left(1-x^{2}\right)^{2} y^{4} \\
& +72\left[x^{2}+2\left(1-x^{2}\right) y\right]\left(1-x^{2}\right)\left(1-y^{2}\right) \\
& +18 x\left[3 x^{2}+\left(6+2 x^{2}\right) y+\left(1-x^{2}\right) y^{2}\right]\left(1-x^{2}\right)\left(1-y^{2}\right) u \\
& +9\left[15-23 x^{2}+\left(24 x^{2}-16\right) y+\left(1-x^{2}\right) y^{2}\right]\left(1-x^{2}\right)\left(1-y^{2}\right) u^{2}
\end{aligned}
$$

for $(x, y, u) \in(0,1)^{3}$.

(a) Suppose that $\varphi_{3}(x, y)=\varphi_{4}(x, y)$ for $x, y \in(0,1)$. Since $\varphi_{1}(x, y)+$ $\varphi_{4}(x, y) \geq 0$ and $\varphi_{2}(x, y) \geq 0$ for $x, y \in(0,1)$, we have 


$$
\begin{aligned}
\Phi(x, y, u) & =\varphi_{1}(x, y)+\varphi_{4}(x, y)+\varphi_{2}(x, y) u \\
& \leq \varphi_{1}(x, y)+\varphi_{4}(x, y)+\varphi_{2}(x, y) \\
& \leq \max \left\{\Phi(x, y, 1): x, y \in[0,1], \varphi_{3}=\varphi_{4}\right\} \\
& \leq \max \{\Phi(x, y, 1): x, y \in[0,1]\} \leq 135 .
\end{aligned}
$$

The last inequality follows from A.3, B.5-8 and C.6.

(b) Suppose that $\varphi_{3}(x, y) \neq \varphi_{4}(x, y)$ for $x, y \in(0,1)$. Then $\partial \Phi / \partial u=0$ if, and only if,

$$
u=\frac{-x\left[3 x^{2}+\left(6+2 x^{2}\right) y+\left(1-x^{2}\right) y^{2}\right]}{15-23 x^{2}+\left(24 x^{2}-16\right) y+\left(1-x^{2}\right) y^{2}}=: u(x, y)
$$

for $(x, y) \in(0,1)^{2}$ such that $\varphi_{3}(x, y) \neq \varphi_{4}(x, y)$. A numerical computation shows that all real and complex solutions of the system of equations $\partial \Phi / \partial x(x, y, u(x, y))=$ 0 and $\partial \Phi / \partial y(x, y, u(x, y))=0$ are the following:

$$
\begin{aligned}
& (x \approx \pm 7.3296, y \approx-3.9586), \quad(x \approx \pm 3.9891, y \approx-2.2430) \\
& (x \approx \pm 0.7783, y \approx-0.9349), \quad(x \approx \pm 2.4991, y \approx 1.1601) \\
& \left(x \approx 4.0332 \cdot 10^{14}, y \approx 108.2175\right), \quad(x \approx \pm 2.6637 \mathrm{i}, y \approx 0.4482) \\
& (x \approx 0.6713 \mathrm{i}, y \approx 0.5624), \quad(x \approx 56781.0229 \mathrm{i}, y \approx 10.8430) \\
& \left(x \approx \pm 2.5455 \cdot 10^{6} \mathrm{i}, y \approx 16.3752\right)
\end{aligned}
$$

Therefore, $\Phi$ has no critical point.

Summarising from parts A to $\mathrm{C}(9)$ follows.

To see that (9) is sharp consider the function $f_{0} \in \mathcal{A}$ given by (10) which belongs $\mathcal{P}^{\prime}$, with $a_{2}=a_{3}=a_{5}=0$ and $a_{4}=-1 / 2$, which completes the proof.

Acknowledgements The authors thank the referees for their careful reading and improvements.

Funding Not applicable.

Availability of data and material Not applicable. The manuscript has no associated data.

Code availability Not applicable.

\section{Declarations}

Conflict of interest The authors declare that they have no conflict of interest.

Open Access This article is licensed under a Creative Commons Attribution 4.0 International License, which permits use, sharing, adaptation, distribution and reproduction in any medium or format, as long as you give appropriate credit to the original author(s) and the source, provide a link to the Creative Commons licence, and indicate if changes were made. The images or other third party material in this article are included in the article's Creative Commons licence, unless indicated otherwise in a credit line to the material. If material is not included in the article's Creative Commons licence and your intended use is not permitted by statutory regulation or exceeds the permitted use, you will need to obtain 
permission directly from the copyright holder. To view a copy of this licence, visit http:// creativecommons.org/licenses/by/4.0/.

\section{References}

1. Alexander, J.W.: Functions which map the interior of the unit circle upon simple regions. Ann. Math. 17(1), 12-22 (1915)

2. Babalola, K.O.: On $H_{3}(1)$ Hankel determinant for some classes of univalent functions. Inequal. Theory Appl. 6, 1-7 (2010)

3. Carathéodory, C.: Über den Variabilitatsbereich der Koeffizienten von Potenzreihen, die gegebene werte nicht annehmen. Math. Ann. 64, 95-115 (1907)

4. Carlitz, L.: Hankel determinants and Bernoulli numbers. Tohoku Math. J. 5, 272-276 (1954)

5. Cho, N.E., Kowalczyk, B., Kwon, O.S., Lecko, A., Sim, Y.J.: The bounds of some determinants for starlike functions of order alpha. Bull. Malays. Math. Sci. Soc. 41, 523-535 (2018)

6. Goodman, A.W.: Univalent Functions. Mariner, Tampa (1983)

7. Hayman, W.K.: On the second Hankel determinant of mean univalent functions. Proc. Lond. Math. Soc. 18(3), 77-94 (1968)

8. Janteng, A., Halim, S.A., Darus, M.: Coefficient inequality for a function whose derivative has a positive real part. J. Inequal. Pure Appl. Math. 7(2), 1-5 (2006). (Art. 50)

9. Janteng, A., Maharana, S., Prajapat, J.K.: Third order Hankel determinant for certain univalent functions. J. Korean Math. Soc. 52(6), 1-5 (2015)

10. Kanas, A., Lecko, A.: On the Fekete-Szegö problem and the domain of convexity for a certain class of univalent functions. Folia Sci. Univ. Tech. Resov. 73, 49-57 (1990)

11. Kowalczyk, B., Lecko, A., Lecko, M., Sim, Y.J.: The sharp bound of the third Hankel determinant for some classes of analytic functions. Bull Korean Math. Soc. 55(6), 1859-1868 (2018)

12. Kowalczyk, B., Lecko, A., Sim, Y.J.: The sharp bound of the Hankel determinant of the third kind for convex functions. Bull. Aust. Math. Soc. 97, 435-445 (2018)

13. Kwon, O.S., Lecko, A., Sim, Y.J.: On the fourth coefficient of functions in the Carathéodory class. Comput. Methods Funct. Theory 18, 307-314 (2018)

14. Lecko, A., Sim, Y.J., Śmiarowska, B.: The sharp bound of the Hankel determinant of the third kind for starlike functions of order 1/2. Complex Anal. Oper. Theory 13, 2231-2238 (2019)

15. Lee, S.K., Ravichandran, V., Supramanian, S.: Bound for the second Hankel determinant of certain univalent functions. J. Inequal. Appl. 2013(281), 1-17 (2013)

16. Libera, R.J., Zlotkiewicz, E.J.: Early coefficients of the inverse of a regular convex function. Proc. Am. Math. Soc. 85(2), 225-230 (1982)

17. Libera, R.J., Zlotkiewicz, E.J.: Coefficient bounds for the inverse of a function with derivatives in $\mathcal{P}$. Proc. Am. Math. Soc. 87(2), 251-257 (1983)

18. MacGregor, T.H.: Functions whose derivative has a positive real part. Trans. Am. Math. Soc. 104(4), 532-537 (1962)

19. Obradović, M., Tuneski, N.: Some properties of the class $\mathcal{U}$. Ann. Univ. Mariae Curie-Skłodowska Sect. A 73, 49-56 (2019)

20. Pommerenke, C.: On the coefficients and Hankel determinants of univalent functions. J. Lond. Math. Soc. 41, 111-122 (1966)

21. Pommerenke, C.: On the Hankel determinants of Univalent functions. Mathematika 14, 108-112 (1967)

22. Pommerenke, C.: Univalent Functions. Vandenhoeck \& Ruprecht, Göttingen (1975)

23. Schoenberg, I.J.: On the maxima of certain Hankel determinants and the zeros of the classical orthogonal polynomials. Indag. Math. 21, 282-290 (1959)

24. Zaprawa, P.: Determinants, third Hankel, for subclasses of univalent functions. Mediterr. J. Math. 14(1), 1-10 (2017). (Art. 19)

Publisher's Note Springer Nature remains neutral with regard to jurisdictional claims in published maps and institutional affiliations. 